\title{
Lower Limb Neuromuscular Modification and Standing Postural Control Alteration in Apparent Asymptomatic People Living with HIV
}

Martín G. Rosario PT, PhD, CSFI, ATRIC*, Jonathan Marshall, SPT, Aaron Herkert, SPT, Bincy Binoy, SPT, Hannah Windham, SPT, Elizabeth Orozco PT, DPT

Physical Therapy Program, Texas Woman's University, Dallas Campus; Texas, United States.

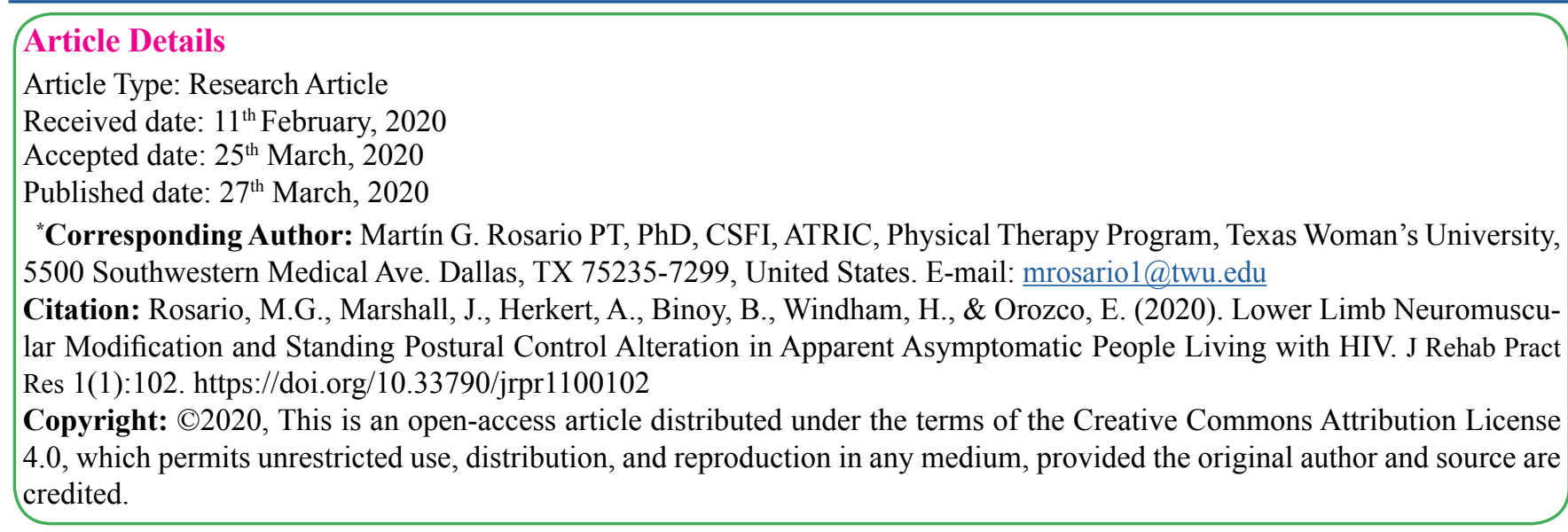

\begin{abstract}
s
Introduction: Balance and gait impairments activation of lower extremity musculature has been shown to be affected in those with HIV. Nevertheless, the precise nature and evidence related to the effects of HIV on neuromuscular activation is limited.

Purpose: This study seeks to evaluate lower extremity neuromuscular activation and postural balance during dual tasks in individuals living with HIV.

Methods: Twenty-four participants of Hispanic-Latino origin diagnosed with HIV (13 male and 11 female, average age $59.2 \pm$ 1.7 years) with an average CD4 count of 612.25 (22 years of HIV diagnosis) enrolled in this study. Thirty-one healthy adults $(7$ male and 24 female, the average age of $25.07 \pm 3.46$ years) were recruited. Surface electromyography (EMG) on the tibialis anterior (TA) and gastrocnemius (GA) muscles were used on the participant's dominant leg with accelerometers/gyroscopes placed on the trunk during balance activities. Each participant was instructed to stand in a bipedal posture on a balance foam for 15 seconds per task. Four single balance and dual cognitive-balance tasks (count backward from 100 in increments of 3 ) were performed on the balance foam.
\end{abstract}

Results: Significant differences $(\mathrm{P}<0.01)$ were found in total Sway, AP/ML Jerk, and AP/ML Distance among groups.

Conclusions: Asymptomatic HIV participants exhibit increased postural instability when compared to controls in all balance tasks. As the GA plays a significant role in static balance, an increased fall risk could be the result of this delay in time to peak onset. As such, our research recommends lower extremity electromyography and strength assessment in this population to forestall or decrease fall hazards.

Keywords: HIV, Postural Instability, ACC.

\section{Introduction}

In the United States, there were 38,739 HIV diagnoses in 2017 with the southern states encompassing $52 \%$ of these new diagnoses [1]. Of the states and dependents within the United States, Puerto Rico has among the highest rates of HIV diagnoses [1]. Balance is achieved by the intricate coordination between three sensory systems: somatosensory, visual, and vestibular, and is subsequently integrated into the central nervous system [2]. In those diagnosed with HIV, the central nervous system has been shown to have some degree of alteration with associated neurocognitive changes [3-5]. Balance and gait impairments may be present in those with HIV and may be influenced by several factors, including time since diagnosis and disease severity $[6,7]$. HIV has also been associated with other diseases and comorbidities. These include dyslipidemia and other metabolic conditions, cardiovascular disease, diabetes mellitus, and polypharmacy [8-12]. Additionally, the activation of lower extremity musculature has also been shown to be affected in those with HIV, especially knee extensors and ankle dorsiflexors, which are important for correcting and maintaining balance [13]. Those with HIV may also have atypical skeletal muscle makeup on a biochemical level and reduced physical function compared to uninfected people [14]. However, the precise nature and effect of HIV on muscle activation is limited. The purpose of this study was to investigate whether there is a difference in postural control and the muscular activation of physical active asymptomatic people diagnosed with HIV compared to younger healthy adults.

\section{Methods}

\section{Participants}

A total of 55 participants (Table 1) between the ages of 18 to 80 years old were given the opportunity to participate in this study. The participants were divided into either HIV group or a control group. The HIV group study was conducted in San Juan Puerto Rico at a Hispanic/Latino Rehabilitation Clinic for HIV patients, called La Perla de Gran Precio. The control group was performed at Texas Woman's University T. Boone Pickens Institute of Health Sciences.

The HIV group consisted 24 participants of Hispanic-latino origin diagnosed with HIV (13 male and 11 female, average age $59.2 \pm$ 1.7 years, average BMI of $25.1 \pm 5.7)$ with an average CD4 count of 612.25 (22 years of HIV diagnosis) and an average ABC score of $72.4 \pm 13.83$ were enrolled in this study. These subjects signed an informed consent, were interviewed, and evaluated at the La Perla de Gran Precio (Community wellness center specialized in individuals with HIV) localized in San Juan, Puerto Rico. The consent form granted access to their medical records, HIV status, CD4+ cell counts and a prerequisite to take part in the study.

The inclusion criteria were: 1) Diagnosed with HIV, 2) CD4 levels above 200 cells/uL, 3) Age within the variety of 25-80 years, 4) Walk without an assistive device, 5) Tolerate the standing position for at least 30 minutes, 6) Stable cardiorespiratory system, 7) Perform 5 time sit to stand test. Since the goal of this study was to identify participants with no apparent balance difficulty, and to establish a baseline 


\begin{tabular}{|l|l|}
\hline \multicolumn{2}{|l|}{ HIV Participants } \\
\hline Characteristics & \multicolumn{2}{l|}{ Study Participants $\mathrm{n}=24$} \\
\hline Age & $\mathrm{M}=59.2+/$ - 1.7 years \\
\hline Gender & Male $=13 ;$ Female $=11$ \\
\hline CD4 & $\mathrm{M}=612.25$ \\
\hline BMI & $\mathrm{M}=25.1+/-5.7$ \\
\hline ABC Scale & $\mathrm{M}=72.4+/-13.83$ \\
\hline Control & \\
\hline Characteristics & Study Participants $\mathrm{n}=31$ \\
\hline Age & $25.07+/-3.46$ years \\
\hline Gender & Male $=7$; Female $=24$ \\
\hline BMI & $\mathrm{M}=23.92$ \\
\hline
\end{tabular}

for standing postural control measurements, the following exclusion criteria was designed: 1) CD4 levels less than 200 cells/uL, 2) Severe balance impairments, 3) Severe visual acuity problems that are not treated, 4) Falls during the last 6 months, 5) Back or lower extremities lesion or surgery during the last 6 months, 6) Use of medications that cause drowsiness 24 hours previous to intervention, and 7) Women that are pregnant or think they might be pregnant.

Each subject was evaluated by performing an interview and reviewing their medical record for the inclusion and exclusion criteria assessment. Medical records were examined by testers to approve the CD4 levels matched the criteria of being above 200 cells/uL. To rule out severe balance impairments, we used the Romberg Test. For the Romberg Test [15], subjects must maintain standing position for 30 seconds with eyes closed. After the screening of the inclusion and exclusion criteria, a total of 24 subjects ( 13 male and 11 female) were able to participate in the study.

For the control group, data collection was performed at Texas Woman's University T. Boone Pickens Institute of Health Sciences (TWU). A total of 31 (7 male and 24 female) healthy participants (average age of $25.07 \pm 3.46$ years, average BMI of 23.92 ) were recruited from the university and the surrounding community to participate in this research study benefitting the advancement of balance interventions in the HIV population. The control participants were recruited via information spread by word of mouth and flyers posted at TWU. The flyer clearly stated that individuals wanting to participate in the control group needed to be HIV negative. However, the control group will go through the same screening and balance protocol as the HIV group. The inclusion criteria, sex, of the nonHIV participants will be comparable to the HIV group.

\section{Measures}

Neuromuscular data was recorded by electromyography (EMG), using a surface electrode system (Delsys, Inc. Boston, MA). The EMG activity of the quadriceps, hamstrings, tibialis anterior, and gastrocnemius muscles were collected at $1,000 \mathrm{~Hz}$ with the electrodes placed according to the recommendations of Sacco \& Kasman. EMG variables included time to peak, decay, duration and amplitude of muscle contraction.

Mobility Lab: APDM's Mobility LabTM (APDM Inc, http://apdm. com) is a transportable gait and balance laboratory designed for the assessment of motion analysis. The mobility lab is a set of sensors that are set in place with belts/straps with the purpose of measuring spatial/temporal gait and balance. This tool is designed to measure kinematics from the tunk, lower and upper extremities. Our variables of interest are anterior-posterior/ medial-lateral distance, velocity, and sway.

\section{Procedures}

The entirety of time commitment for the participant will consist of 30 minutes for testing/screening protocol and 30-45 $\mathrm{min}$ for balance testing protocol, for a maximum total time of 1.5 hours in one session. After completing an assessment and receiving consent, participants were asked to identify their dominant leg. This was done by asking each individual which leg they would use to kick a soccer ball. If necessary, areas of the dominant leg were shaved with a non-electric razor in order to attach and secure the placement of the various Electromyography (EMG) sensors. Electromyography (EMG) surface electrodes were placed on their dominant leg over the quadriceps (QUAD), hamstrings (HAM), anterior tibialis (TA), and gastrocnemius (GA). Each participant wore Mobility Lab sensors around bilateral wrist and ankle as well as their chest and lower back.

The participant was asked to perform a maximal contraction test for each muscle group. Maximum contraction for the quadriceps muscle was tested by asking the participant to sit in a chair with a gait belt surrounding the legs of the chair. The participant placed their dominant leg posterior to the gait belt and asked to extend the leg into belt with full force, holding the contraction. For the hamstrings, the participant remained in the same chair but was asked to place their leg anterior to the gait belt and to perform a knee flexion force into belt. The anterior tibialis was tested while the participant stood supported with a hand on the chair, heels on the ground, and toes in the air while a tester applied a resistive force distal to the foot. The gastrocnemius was tested by having the patient lift their heel off the ground and onto their toes while pushing into the ground. Each of the positions were held for 10 seconds as the participant held a maximal contraction force. The EMG surface electrodes and Mobility Lab sensors were worn for the entirety of the participants. The five times sit to stand test was also administered to all participants in order to quantify functional lower extremity strength and/or identify movement strategies a patient uses to complete transitional movements. The subject will be seated in a static chair with a backrest, no arm rest, and also placed near a wall to prevent backward displacement of the chair during the test. The evaluators will indicate the subject to sit and [16] stand five times as fast as possible and will record the time it takes to complete the test. For this study, we expect our subjects to do this task in 10 seconds or less. Persons less than 60 years of age that requires more than 10 seconds to complete the task could present balance disturbances due to decreased functional strength in the lower extremities. If the subject cannot complete the task in the required time they will be excluded from the study.

\section{Balance Assessment}

Balance was measured not only using instrumented (mobility lab accelerometers) but also a clinical test (ABC scale). After signing the 
informed consent and collecting demographic data, participants completed the activity-specific balance confidence (ABC) scale. A member of the research team placed a lumbar accelerometer on each subject. Each participant was instructed to stand in a static bi-pedal posture on a firm surface or a thick foam pad (having the mobility lab belt accelerometers sensors on) and perform eight balance tasks. Data of anterior-posterior sway, medial- lateral sway, and sway were collected in each of the conditions. Each task took 15 seconds to be performed, plus the 2 minutes of rest between every two tasks. The first task was performed on a foam pad surface with eyes open, representing the baseline for this study. Balance task were divided into four single and four dual cognitive tasks.

First component of the balance assessment (measured balance) included one tasks on firm surface and four tasks performed on a thick foam pad on a hard surface of the floor. These five tasks were considered the single tasks and are depicted as follows: 1-2) Eyes open looking at a fixed point on the wall to examine all systems on a firm surface (all systems unaltered) and on a thick foam pad to (alter somatosensory) (EO); 3) Eyes closed on a thick foam pad (with the head fixed approximately 90 degrees) to alter somatosensory and cancel out the visual input (EC); 4) Eyes open looking at a fixed point on the wall and actively moving head upward and downward to alter vestibular inputs (The subject was instructed to maintain the movement frequency of $60 \mathrm{bpm}$ with a metronome in the room and amplitude of approximately 45 degrees in each direction: neck flexion and extension; 5) Eyes closed (with head fixed approximately 90 degrees) to cancel out visual input and active upward and downward head movement to modify vestibular inputs (EC+HUD). The subject is instructed to maintain the movement frequency and amplitude as described in previous task.

The subjects were asked to perform four more tasks, continuing to stand on a thick foam pad placed on a firm surface. The remaining four tasks were like the aforementioned 2-5 tasks. Nonetheless, to add the dual cognitive task components of the balance assessment we asked participants to count backwards by threes. Since neurocognitive impairments are evident at some stages of the diseases we added the cognitive dual task to the balance test to divide attention and identify alterations that potentially can affect motor control in these individuals.
The second part of our assessment included the perception of balance (balance perception) or confidence among different activities. We used the ABC scale [17] for this purpose. The ABC scale is a 16-item questionnaire pertaining to various activities that require maintaining balance to achieve. Participants rate each item on a scale from zero percent to 100 percent. A rating of zero percent indicates no confidence in maintaining balance during the activity, while 100 percent indicates full confidence that they would maintain their balance. The activities vary in difficulty and cognitive requirement.

\section{Results}

The variables of interest in this study were 1) time to peak, 2) decay and, 3) duration of muscle activation for TA and GA. A repeated measure MANOVA analysis was used to compare all variables of interest. Table $2 \& 3$ show no significant difference is indicated between duration and decay of muscle activation for TA and GA across the various tasks assessed. Table $2 \& 3$ also show no significant difference between asymptomatic HIV group and control group in Electromyographic (EMG) amplitude or percent of task for amplitude. Next we analyzed 1) total Sway $\left.\left(\mathrm{m}^{2} / \mathrm{s}^{4}\right), 2\right)$ AP Jerk $\left(\mathrm{m}^{2} /\right.$ $\left.\mathrm{s}^{5}\right)$ and ML Jerk $\left(\mathrm{m}^{2} / \mathrm{s}^{5}\right)$, and 3) AP Distance $\left(\mathrm{m} / \mathrm{s}^{2}\right)$, ML Distance $\left(\mathrm{m} / \mathrm{s}^{2}\right)$ and AP Velocity $(\mathrm{m} / \mathrm{s})$, ML Velocity $(\mathrm{m} / \mathrm{s})$. A non-parametric Wilcoxon Signed Ranks test was performed to compare the variables between control and HIV participants. According to Table 4, HIV group swayed significantly more during all balance tasks. Table $5 \& 6$ showed that HIV participants moved significantly further $(\mathrm{P}<.001)$ in both the anteroposterior and medial jerk. AP and ML distance was also significantly increased $(\mathrm{P}<.001)$ in the HIV group when compared to the controls as shown in Tables $7 \& 8$. According to Table 9, HIV participants performed significantly slower in the AP velocity during EO COG $(\mathrm{P}<.001)$ and EO firm $(\mathrm{P}<.01)$. However, the HIV participants moved significantly faster during the eyes closed, head up and down task while counting backwards $(\mathrm{P}<.025)$. Table 10 stated that EO COG was the only task that the HIV group performed significantly slower $(\mathrm{P}<.025)$ in $\mathrm{ML}$ velocity. No significant differences were found in the remaining tasks when comparing the velocity component of sway.

\begin{tabular}{|l|l|l|l|l|}
\hline Task & Variables & $\begin{array}{l}\text { Control } \\
(\mathrm{N}=19)\end{array}$ & $\begin{array}{l}\text { Asymptomatic HIV } \\
(\mathrm{N}=18)\end{array}$ & P-value \\
\hline \multirow{5}{*}{ EO Firm } & Gastrocnemius & & & \\
& Amplitude* & $27.94 \pm 17.75$ & $19.44 \pm 13.79$ & .081 \\
\cline { 2 - 5 } & Amplitude \%** & $33.81 \pm 32.36$ & $40.04 \pm 29.75$ & .500 \\
\cline { 2 - 5 } & Time to Peak (sec) & $.33 \pm .14$ & $.37 \pm .20$ & .568 \\
\cline { 2 - 5 } & Duration (sec) & $.73 \pm .18$ & $.73 \pm .29$ & .979 \\
\cline { 2 - 5 } & Decay (sec) & $.39 \pm .12$ & $.37 \pm .19$ & .545 \\
\hline \multirow{5}{*}{ EO Foam } & Amplitude* & $33.26 \pm 24.95$ & $27.29 \pm 15.99$ & .357 \\
\cline { 2 - 5 } & Amplitude \%** & $36.38 \pm 28.09$ & $47.78 \pm 27.90$ & .169 \\
\cline { 2 - 5 } & Time to Peak (sec) & $.34 \pm .13$ & $.38 \pm .17$ & .490 \\
\cline { 2 - 5 } & Duration (sec) & $.70 \pm .14$ & $.72 \pm .16$ & .693 \\
\cline { 2 - 5 } & Decay (sec) & $.35 \pm .12$ & $.34 \pm .14$ & .742 \\
\hline \multirow{5}{*}{ EC Foam } & Amplitude* & $41.59 \pm 22.74$ & $32.46 \pm 13.34$ & .119 \\
\cline { 2 - 5 } & Amplitude \%** & $37.40 \pm 28.52$ & $44.52 \pm 30.80$ & .410 \\
\cline { 2 - 5 } & Time to Peak (sec) & $.38 \pm .16$ & $.34 \pm .14$ & .424 \\
\cline { 2 - 5 } & Duration (sec) & $.71 \pm .21$ & $.77 \pm .21$ & .431 \\
\cline { 2 - 5 } & Decay (sec) & $.33 \pm .14$ & $.42 \pm .19$ & .094 \\
\hline
\end{tabular}

Table. 2 To be Cont... 


\begin{tabular}{|c|c|c|c|c|}
\hline \multirow[t]{5}{*}{ EO HUD } & Amplitude* & $32.13 \pm 20.24$ & $38.16 \pm 22.90$ & .336 \\
\hline & Amplitude $\% * *$ & $36.67 \pm 27.95$ & $51.20 \pm 30.92$ & .093 \\
\hline & Time to Peak (sec) & $.36 \pm .16$ & $.41 \pm .14$ & .354 \\
\hline & Duration (sec) & $.70 \pm .24$ & $.77 \pm .16$ & .753 \\
\hline & Decay $(\mathrm{sec})$ & $.34 \pm .17$ & $.35 \pm .09$ & .368 \\
\hline \multirow[t]{5}{*}{ EC HUD } & Amplitude* & $41.69 \pm 25.07$ & $46.97 \pm 22.09$ & .454 \\
\hline & Amplitude $\% * *$ & $34.65 \pm 25.54$ & $49.27 \pm 30.36$ & .074 \\
\hline & Time to Peak (sec) & $.32 \pm .16$ & $.35 \pm .20$ & .519 \\
\hline & Duration $(\mathrm{sec})$ & $.67 \pm .19$ & $.77 \pm .22$ & .179 \\
\hline & Decay (sec) & $.35 \pm .12$ & $.41 \pm .16$ & .261 \\
\hline \multirow[t]{5}{*}{ EO COG } & Amplitude* & $36.23 \pm 23.51$ & $24.17 \pm 15.49$ & .053 \\
\hline & Amplitude \%** & $48.11 \pm 30.71$ & $32.68 \pm 24.06$ & .333 \\
\hline & Time to Peak (sec) & $.31 \pm .14$ & $.47 \pm .20$ & .008 \\
\hline & Duration (sec) & $.63 \pm .17$ & $.85 \pm .27$ & .008 \\
\hline & Decay (sec) & $.31 \pm .12$ & $.37 \pm .17$ & .264 \\
\hline \multirow[t]{5}{*}{ EC COG } & Amplitude* & $34.01 \pm 15.93$ & $32.68 \pm 14.33$ & .769 \\
\hline & Amplitude \%** & $46.59 \pm 30.15$ & $49.17 \pm 30.81$ & .772 \\
\hline & Time to Peak (sec) & $.32 \pm .12$ & $.32 \pm .14$ & .979 \\
\hline & Duration (sec) & $.67 \pm .16$ & $.71 \pm .20$ & .528 \\
\hline & Decay (sec) & $.34 \pm .11$ & $.38 \pm .18$ & .421 \\
\hline \multirow[t]{5}{*}{ EO HUD COG } & Amplitude* & $37.06 \pm 20.46$ & $33.41 \pm 20.03$ & .539 \\
\hline & Amplitude \%** & $42.90 \pm 33.89$ & $37.25 \pm 29.58$ & .552 \\
\hline & Time to Peak (sec) & $.32 \pm .13$ & $.29 \pm .18$ & .645 \\
\hline & Duration (sec) & $.68 \pm .19$ & $.71 \pm .18$ & .373 \\
\hline & Decay $(\mathrm{sec})$ & $.36 \pm .14$ & $.41 \pm .20$ & .667 \\
\hline \multirow[t]{5}{*}{ EC HUD COG } & Amplitude* & $34.93 \pm 18.00$ & $41.92 \pm 18.94$ & .197 \\
\hline & Amplitude \%** & $44.11 \pm 33.11$ & $54.50 \pm 31.54$ & .279 \\
\hline & Time to Peak (sec) & $7.49 \pm .99$ & $7.67 \pm .66$ & .538 \\
\hline & Duration (sec) & $.35 \pm .10$ & $.44 \pm .15$ & .366 \\
\hline & Decay (sec) & $.39 \pm .14$ & $.35 \pm .15$ & .049 \\
\hline
\end{tabular}

Table 2: Electromyographic (EMG) amplitude, percent (\%) of task for amplitude, time to peak, duration, and decay of gastrocnemius during balance tasks. Results of multivariate analysis of variance (MANOVA) performed between asymptomatic HIV group and control group. Significance level set at $\mathrm{p} \leq 0.05$.

\begin{tabular}{|l|l|l|l|l|}
\hline Task & Variables & Control (N = 19) & Asymptomatic HIV (N = 18) & P-value \\
\hline \multirow{4}{*}{ EO Firm } & Tibialis Anterior & & & \\
& Amplitude* & $43.25 \pm 33.15$ & $35.29 \pm 18.55$ & .346 \\
\cline { 2 - 5 } & Amplitude \%** & $43.59 \pm 28.84$ & $46.40 \pm 31.37$ & .751 \\
\cline { 2 - 5 } & Time to Peak (sec) & $.36 \pm .14$ & $.33 \pm .15$ & .530 \\
\cline { 2 - 5 } & Duration (sec) & $.72 \pm .17$ & $.79 \pm .28$ & .335 \\
\cline { 2 - 5 } & Decay (sec) & $.36 \pm .13$ & $.46 \pm .26$ & .137 \\
\hline \multirow{5}{*}{ EO Foam } & Amplitude* & $39.16 \pm 21.31$ & $39.78 \pm 21.20$ & .921 \\
\cline { 2 - 5 } & Amplitude \%** & $48.08 \pm 23.84$ & $48.21 \pm 32.46$ & .987 \\
\cline { 2 - 5 } & Time to Peak (sec) & $.33 \pm .16$ & $.32 \pm .13$ & .830 \\
\cline { 2 - 5 } & Duration (sec) & $.70 \pm .18$ & $.71 \pm .29$ & .878 \\
\cline { 2 - 5 } & Decay (sec) & $.37 \pm .14$ & $.39 \pm .25$ & .741 \\
\hline
\end{tabular}

Table. 3 To be Cont....... 


\begin{tabular}{|c|c|c|c|c|}
\hline \multirow[t]{5}{*}{ EC Foam } & Amplitude* & $44.19 \pm 24.91$ & $55.92 \pm 26.54$ & .127 \\
\hline & Amplitude $\% * *$ & $41.42 \pm 26.96$ & $38.91 \pm 26.95$ & .754 \\
\hline & Time to Peak (sec) & $.34 \pm .17$ & $.43 \pm .29$ & .257 \\
\hline & Duration (sec) & $.75 \pm .21$ & $.82 \pm .35$ & .442 \\
\hline & Decay (sec) & $.40 \pm .12$ & $.38 \pm .27$ & .804 \\
\hline \multirow[t]{5}{*}{ EO HUD } & Amplitude* & $45.88 \pm 22.33$ & $54.83 \pm 22.80$ & .185 \\
\hline & Amplitude \%** & $45.07 \pm 24.84$ & $57.82 \pm 36.52$ & .156 \\
\hline & Time to Peak (sec) & $.34 \pm .19$ & $.36 \pm .19$ & .749 \\
\hline & Duration (sec) & $.70 \pm .25$ & $.64 \pm .59$ & .699 \\
\hline & Decay (sec) & $.35 \pm .14$ & $.27 \pm .64$ & .612 \\
\hline \multirow[t]{5}{*}{ EC HUD } & Amplitude* & $43.86 \pm 23.19$ & $66.90 \pm 25.30$ & .002 \\
\hline & Amplitude $\% * *$ & $47.37 \pm 28.34$ & $48.79 \pm 30.54$ & .869 \\
\hline & Time to Peak (sec) & $.35 \pm .13$ & $.37 \pm .17$ & .793 \\
\hline & Duration (sec) & $.65 \pm .21$ & $.68 \pm .24$ & .699 \\
\hline & Decay (sec) & $.30 \pm .12$ & $.31 \pm .11$ & .694 \\
\hline \multirow[t]{5}{*}{ EO COG } & Amplitude* & $42.42 \pm 21.94$ & $37.60 \pm 18.66$ & .435 \\
\hline & Amplitude $\% * *$ & $43.45 \pm 25.06$ & $48.83 \pm 29.96$ & .504 \\
\hline & Time to Peak (sec) & $.33 \pm .13$ & $.31 \pm .12$ & .756 \\
\hline & Duration (sec) & $.64 \pm .19$ & $.72 \pm .25$ & .259 \\
\hline & Decay (sec) & $.31 \pm .10$ & $.40 \pm .21$ & .081 \\
\hline \multirow[t]{5}{*}{ EC COG } & Amplitude* & $41.20 \pm 23.31$ & $47.46 \pm 25.07$ & .382 \\
\hline & Amplitude $\% * *$ & $45.00 \pm 25.16$ & $33.36 \pm 26.54$ & .132 \\
\hline & Time to Peak (sec) & $.35 \pm .16$ & $.32 \pm .16$ & .695 \\
\hline & Duration (sec) & $.68 \pm .19$ & $.65 \pm .21$ & .712 \\
\hline & Decay (sec) & $.33 \pm .12$ & $.33 \pm .10$ & .915 \\
\hline \multirow[t]{5}{*}{ EO HUD COG } & Amplitude* & $47.15 \pm 21.75$ & $48.10 \pm 22.48$ & .885 \\
\hline & Amplitude $\% * *$ & $48.14 \pm 27.70$ & $38.12 \pm 27.98$ & .228 \\
\hline & Time to Peak (sec) & $.30 \pm .07$ & $.35 \pm .12$ & .119 \\
\hline & Duration (sec) & $.59 \pm .13$ & $.64 \pm .15$ & .267 \\
\hline & Decay (sec) & $.29 \pm .12$ & $.29 \pm .10$ & .992 \\
\hline \multirow[t]{5}{*}{ EC HUD COG } & Amplitude* & $45.34 \pm 20.85$ & $59.84 \pm 24.59$ & .033 \\
\hline & Amplitude \%** & $43.95 \pm 30.84$ & $36.49 \pm 27.95$ & .400 \\
\hline & Time to Peak (sec) & $.35 \pm .13$ & $.30 \pm .13$ & .291 \\
\hline & Duration (sec) & $.70 \pm .18$ & $.67 \pm .23$ & .683 \\
\hline & Decay (sec) & $.35 \pm .15$ & $.36 \pm .17$ & .738 \\
\hline
\end{tabular}

Table 3: Electromyographic (EMG) amplitude, percent (\%) of task for amplitude, time to peak, duration, and decay of tibialis anterior during balance tasks. Results of multivariate analysis of variance (MANOVA) performed between asymptomatic HIV group and control group. Significance level set at $\mathrm{p} \leq 0.05$.

\begin{tabular}{|l|l|l|l|}
\hline \multicolumn{2}{|l|}{ Total Sway $\left(\mathrm{m}^{2} / \mathrm{s}^{4}\right)$} \\
\hline Task & Control Group & HIV Group & P-value \\
\hline EO Firm & $.008 \pm .016$ & $1.20 \pm 3.14$ & 0.01 \\
\hline EO Foam & $.032 \pm .080$ & $4.05 \pm 17.00$ & 0.01 \\
\hline EC Foam & $.031 \pm .063$ & $9.75 \pm 31.57$ & 0.01 \\
\hline EO HUD & $.018 \pm .008$ & $3.95 \pm 8.83$ & 0.01 \\
\hline EC HUD & $.047 \pm .050$ & $21.47 \pm 69.60$ & 0.01 \\
\hline EO COG & $.032 \pm .037$ & $4.08 \pm 13.25$ & 0.01 \\
\hline
\end{tabular}




\begin{tabular}{|l|l|l|l|}
\hline EC COG & $.037 \pm .062$ & $6.77 \pm 21.80$ & 0.01 \\
\hline EO HUD COG & $.051 \pm .100$ & $6.74 \pm 12.19$ & 0.01 \\
\hline EC HUD COG & $.047 \pm .043$ & $13.87 \pm 40.13$ & 0.01 \\
\hline EO= Eyes Open, EC=Eyes Closed, HUD= Head up and down, COG = cognitive task counting backwards from 100 \\
\hline
\end{tabular}

Table 4: Total Sway during balance tasks. Results of non-parametric test (Wilcoxon Signed Rank Test) performed between asymptomatic HIV group and control group. Significance level set at $\mathrm{p} \leq 0.05$.

\begin{tabular}{|l|l|l|l|}
\hline \multicolumn{4}{|l|}{ AP Jerk $\left(\mathrm{m}^{2} / \mathrm{s}^{5}\right)$} \\
\hline Task & Control Group & HIV Group & P-value \\
\hline EO Firm & $.016 \pm .062$ & $3.58 \pm 15.57$ & .000 \\
\hline EO Foam & $.024 \pm .042$ & $9.33 \pm 53.83$ & .000 \\
\hline EC Foam & $.037 \pm .085$ & $15.00 \pm 58.45$ & .000 \\
\hline EO HUD & $.038 \pm .025$ & $12.65 \pm 48.53$ & .000 \\
\hline EC HUD & $.093 \pm .117$ & $28.55 \pm 99.50$ & .000 \\
\hline EO COG & $.067 \pm .079$ & $8.72 \pm 32.33$ & .000 \\
\hline EC COG & $.075 \pm .205$ & $9.59 \pm 23.27$ & .000 \\
\hline EO HUD COG & $.132 \pm .195$ & $19.06 \pm 57.72$ & .000 \\
\hline EC HUD COG & $.103 \pm .116$ & $30.77 \pm 88.04$ & .000 \\
\hline EO= Eyes Open, EC=Eyes Closed HUD= Head up and down COG $=$ cognitive task counting backwards from 100 \\
\hline
\end{tabular}

$\mathrm{EO}=$ Eyes Open, $\mathrm{EC}=$ Eyes Closed, $\mathrm{HUD}=$ Head up and down, $\mathrm{COG}=$ cognitive task counting backwards from 100

Table 5: Anterior-posterior Jerk during balance tasks. Results of non-parametric test (Wilcoxon Signed Rank Test) performed between asymptomatic HIV group and control group. Significance level set at $p \leq 0.05$.

\begin{tabular}{|l|l|l|l|}
\hline \multicolumn{4}{|l|}{ ML Jerk $\left(\mathrm{m}^{2} / \mathrm{s}^{5}\right)$} \\
\hline Task & Control Group & HIV Group & P-value \\
\hline EO Firm & $.007 \pm .023$ & $211.82 \pm 410.15$ & .000 \\
\hline EO Foam & $.056 \pm .168$ & $230.45 \pm 425.58$ & .000 \\
\hline EC Foam & $.031 \pm .074$ & $241.51 \pm 449.41$ & .000 \\
\hline EO HUD & $.009 \pm .005$ & $215.75 \pm 409.24$ & .000 \\
\hline EC HUD & $.032 \pm .047$ & $230.22 \pm 410.81$ & .000 \\
\hline EO COG & $.022 \pm .023$ & $230.94 \pm 424.15$ & .000 \\
\hline EC COG & $.035 \pm .068$ & $228.96 \pm 419.05$ & .000 \\
\hline EO HUD COG & $.023 \pm .025$ & $220.25 \pm 409.40$ & .000 \\
\hline EC HUD COG & $.035 \pm .050$ & $222.81 \pm 409.00$ & .000 \\
\hline EO $=$ Eyes Open, EC=Eyes Closed, HUD= Head up and down, COG $=$ cognitive task counting backwards from 100 \\
\hline
\end{tabular}

Table 6: Medial-lateral Jerk during balance tasks. Results of non-parametric test (Wilcoxon Signed Rank Test) performed between asymptomatic HIV group and control group. Significance level set at $\mathrm{p} \leq 0.05$.

\begin{tabular}{|l|l|l|l|}
\hline \multicolumn{2}{|c|}{ AP Distance $\left(\mathrm{m} / \mathrm{s}^{2}\right)$} \\
\hline Task & Control Group & HIV Group & P-value \\
\hline EO Firm & $.062 \pm .042$ & $3.93 \pm 8.30$ & .000 \\
\hline EO Foam & $.067 \pm .039$ & $5.47 \pm 15.81$ & .000 \\
\hline EC Foam & $.087 \pm .036$ & $8.59 \pm 17.60$ & .000 \\
\hline EO HUD & $.117 \pm .044$ & $9.47 \pm 16.21$ & .000 \\
\hline EC HUD & $.153 \pm .057$ & $16.32 \pm 30.71$ & .000 \\
\hline EO COG & $.115 \pm .090$ & $7.41 \pm 13.20$ & .001 \\
\hline EC COG & $.106 \pm .067$ & $12.39 \pm 29.28$ & .000 \\
\hline EO HUD COG & $.169 \pm .142$ & $12.26 \pm 19.34$ & .000 \\
\hline EC HUD COG & $.147 \pm .057$ & $15.86 \pm 25.89$ & .000 \\
\hline EO Eyes Open EC Ey & Close & \\
\hline
\end{tabular}

$\mathrm{EO}=$ Eyes Open, $\mathrm{EC}=$ Eyes Closed, $\mathrm{HUD}=$ Head up and down, $\mathrm{COG}=$ cognitive task counting backwards from 100

Table 7: Anterior-posterior distance during balance tasks. Results of non-parametric test (Wilcoxon Signed Rank Test) performed between asymptomatic HIV group and control group. Significance level set at $\mathrm{p} \leq 0.05$. 


\begin{tabular}{|l|l|l|l|}
\hline \multicolumn{2}{|l|}{ ML Distance $\left(\mathrm{m} / \mathrm{s}^{2}\right)$} \\
\hline Task & Control Group & HIV Group & P-value \\
\hline EO Firm & $.022 \pm .012$ & $2.67 \pm 4.55$ & .000 \\
\hline EO Foam & $.050 \pm .028$ & $3.02 \pm 4.30$ & .000 \\
\hline EC Foam & $.058 \pm .021$ & $9.85 \pm 34.47$ & .000 \\
\hline EO HUD & $.043 \pm .010$ & $5.44 \pm 12.37$ & .000 \\
\hline EC HUD & $.066 \pm .028$ & $11.41 \pm 29.10$ & .000 \\
\hline EO COG & $.055 \pm .027$ & $8.19 \pm 26.92$ & .000 \\
\hline EC COG & $.059 \pm .031$ & $9.26 \pm 25.94$ & .000 \\
\hline EO HUD COG & $.060 \pm .044$ & $7.86 \pm 18.06$ & .000 \\
\hline EC HUD COG & $.065 \pm .030$ & $9.37 \pm 21.78$ & .000 \\
\hline EO= Eyes Open, EC=Eyes Closed, HUD= Head up and down, COG $=$ cognitive task counting backwards from 100 \\
\hline
\end{tabular}

Table 8: Medial-lateral distance during balance tasks. Results of non-parametric test (Wilcoxon Signed Rank Test) performed between asymptomatic HIV group and control group. Significance level set at $p \leq 0.05$.

\begin{tabular}{|l|l|l|l|}
\hline \multicolumn{4}{|l|}{ AP Velocity $(\mathrm{m} / \mathrm{s})$} \\
\hline Task & Control Group & HIV Group & P-value \\
\hline EO Firm & $.118 \pm .105$ & $.081 \pm .063$ & .009 \\
\hline EO Foam & $.118 \pm .095$ & $.110 \pm .078$ & .591 \\
\hline EC Foam & $.135 \pm .104$ & $.203 \pm .246$ & .225 \\
\hline EO HUD & $.145 \pm .123$ & $.177 \pm .125$ & .700 \\
\hline EC HUD & $.203 \pm .169$ & $.260 \pm .254$ & .536 \\
\hline EO COG & $.216 \pm .281$ & $.131 \pm .116$ & .000 \\
\hline EC COG & $.160 \pm .139$ & $.186 \pm .238$ & .763 \\
\hline EO HUD COG & $0.221 \pm .320$ & $.194 \pm .207$ & .690 \\
\hline EC HUD COG & $0.158 \pm .100$ & $.240 \pm .210$ & .024 \\
\hline EO $=$ Eyes Open, EC=Eyes Closed, HUD= Head up and down, COG = cognitive task counting backwards from 100 \\
\hline
\end{tabular}

Table 9: Anterior-posterior velocity during balance tasks. Results of non-parametric test (Wilcoxon Signed Rank Test) performed between asymptomatic HIV group and control group. Significance level set at $p \leq 0.05$.

\begin{tabular}{|l|l|l|l|}
\hline \multicolumn{2}{|l|}{ ML Velocity (m/s) } \\
\hline Task & Control Group & HIV Group & P-value \\
\hline EO Firm & $.032 \pm .023$ & $.032 \pm .024$ & .879 \\
\hline EO Foam & $.060 \pm .041$ & $.064 \pm .050$ & .935 \\
\hline EC Foam & $.064 \pm .037$ & $.069 \pm .065$ & .454 \\
\hline EO HUD & $.056 \pm .033$ & $.079 \pm .075$ & .199 \\
\hline EC HUD & $.062 \pm .038$ & $.119 \pm .205$ & .035 \\
\hline EO COG & $.073 \pm .050$ & $.059 \pm .048$ & 019 \\
\hline EC COG & $.061 \pm .050$ & $.069 \pm .052$ & .293 \\
\hline EO HUD COG & $.083 \pm .112$ & $.074 \pm .050$ & .847 \\
\hline EC HUD COG & $.067 \pm .033$ & $.084 \pm .057$ & .385 \\
\hline EO= Eyes Open, EC=Eyes Closed, HUD= Head up and down, COG = cognitive task counting backwards from 100 \\
\hline
\end{tabular}

\section{Discussion}

The purpose of this study was to investigate whether there is a difference in muscular activation and balance alteration in physically active people living with HIV compared to healthy adults. Our study focuses on a multi-balance task with a cognitive component to identify postural and neuromuscular alterations in HIV diagnosed participants compared to control.
Postural Balance: Our study found that people living with asymptomatic HIV have increased multi-directional postural sway and reduced balance compared to healthy adults. This is consistent with one meta-analysis that shows balance impairments in people with HIV that resemble fall risk characteristics in non-HIV older adults [6]. Prior studies have also shown an increased number of fall risk factors and the number of falls in those living with HIV compared to uninfected adults [18]. Another study found pontocerebellar changes 
in those with HIV that contributed to postural instability and balance deficits [19]. This aforementioned helps support our findings of multi-directional balance impairment, despite HIV participants being asymptomatic. This multi-directional postural instability has clinical importance when treating patients with asymptomatic HIV. Balance and falls assessments should be performed and interventions should be implemented in a preventative manner that targets and improves balance before overt balance deficits present themselves and potentially lead to falls. The performance of balance and falls assessments with HIV patients has also been proposed by other researchers [20].

Neuromuscular Control and Balance: There is a wide range of neuromuscular diseases and impairments associated with HIV that can affect the musculature and peripheral nerves of the body [21]. Previous studies have found impaired neuromuscular control and motor impairments in those living with HIV as well [13, 22]. We assessed the neuromuscular activation of the TA and GA in healthy adults and in those living with asymptomatic HIV. No significant difference in activation was seen in amplitude, duration, time-topeak or decay for all tasks between groups. A similar study that looked at balance in people living with HIV found that early stages of HIV had postural instability, but they did not see a significant correlation with EMG findings [23]. This may help explain why we found postural instability in our HIV participants, but no significance when looking at neuromuscular activation. Another explanation for this finding is that the recruited asymptomatic HIV participants were actively exercising at a community clinic. Exercise has been shown to mitigate the effects of the reduction in efferent drive and may have counteract losses in neuromuscular activation [24]. A trend was seen in the asymptomatic HIV group towards longer duration of muscle activation during more difficult, dual-task activities. Individuals with HIV have been found to have decreased dual-task performance [25]. Within the duration of muscle activation, we again found a trend with HIV participants taking longer to reach peak muscle contraction. This suggests that those with HIV may have slower muscle activation compared to healthy adults. Clinically, this may manifest when performing actions that require quick reactions such as transitioning from one surface to another during gait.

\section{Conclusion}

This study found static postural alteration in a primarily in a sagittal direction in people living with HIV. Future studies should look at dynamic activities such as walking on different surfaces. Dynamic activities are more functional in nature than static posture, which was assessed in this study. Musculature activation and timing in physically active HIV diagnosed individuals is not a factor affecting balance. This study's results suggest that regular exercise can mitigate some of the neuromuscular alterations that are known to affect these individuals. However, neuromuscular control assessment is recommended further up the kinetic chain, such as at the knee, hip, or trunk, to ascertain the origin previously mentioned balance alteration.

Funding: Not Applicable.

Conflicts of interest/Competing interests: Authors report no conflict or competing interest.

Ethics approval: IRB approval TWU protocol \# 20092.

Authors' contributions: All authors contributed to the study conception and design.

\section{References}

1. Centers for Disease Control and Prevention. Diagnoses of HIV infection in the United States and dependent areas, 2017. 2018;

2. Peterka, R. (2002). Sensorimotor integration in human postural control. Journal of Neurophysiology., 88(3), 1097-1118.

3. Calcagno, A., Perri, G. D., \& Bonora, S. (2017). Treating HIV Infection in the Central Nervous System. Drugs, 77(2), 145-157. doi:10.1007/s40265-016-0678-9
4. Saylor, D., Dickens, A. M., Sacktor, N., Haughey, N., Slusher, B., Pletnikov, M., ... Mcarthur, J. C. (2016). HIV-associated neurocognitive disorder - pathogenesis and prospects for treatment. Nature Reviews Neurology, 12(4), 234-248. doi: 10.1038/nrneurol.2016.27

5. Clifford, D. B., \& Ances, B. M. (2013). HIV-associated neurocognitive disorder. The Lancet Infectious Diseases, 13(11), 976-986. doi: 10.1016/s1473-3099(13)70269-x

6. Berner, K., Morris, L., Baumeister, J., \& Louw, Q. (2017). Objective impairments of gait and balance in adults living with HIV-1 infection: a systematic review and meta-analysis of observational studies. BMC Musculoskeletal Disorders, 18(1), 325.

7. Richert, L., Dehail, P., Mercié, P., Dauchy, F.-A., Bruyand, M., Greib, C., ... Chêne, G. (2011). High frequency of poor locomotor performance in HIV-infected patients. Aids, 25(6), 797-805. doi: 10.1097/qad.0b013e3283455dff

8. Serrão, R., Piñero, C., Velez, J., Coutinho, D., Maltez, F., Lino, S., ... Pássaro, L. (2019). Non-AIDS-related comorbidities in people living with HIV-1 aged 50 years and older: The AGING POSITIVE study. International Journal of Infectious Diseases, 79, 94-100. doi: 10.1016/j.ijid.2018.10.011

9. Maggi, P., Santoro, C. R., Nofri, M., Ricci, E., Gennaro, N. D., Bellacosa, C., ... Socio, G. V. D. (2019). Clusterization of comorbidities and multi-morbidities among persons living with HIV: a cross-sectional study. BMC Infectious Diseases, 19(1). doi: 10.1186/s12879-019-4184-z

10. Mata-Marín, J. A., Martínez-Osio, M. H., Arroyo-Anduiza, C. I., Berrospe-Silva, M. D. L. Á., Chaparro-Sánchez, A., CruzGrajales, I., ... Jerónimo-Morales, M. (2019). Comorbidities and polypharmacy among HIV-positive patients aged 50 years and over: a case-control study. BMC Research Notes, 12(1). doi: 10.1186/s13104-019-4576-6

11. Ghosn, J., Taiwo, B., Seedat, S., Autran, B., \& Katlama, C. (2018). Hiv. The Lancet, 392(10148), 685-697. doi: 10.1016/ s0140-6736(18)31311-4

12. Wu, P.-Y., Chen, M.-Y., Hsieh, S.-M., Sun, H.-Y., Tsai, M.-S., Lee, K.-Y., ... Hung, C.-C. (2014). Comorbidities among the HIV-Infected Patients Aged 40 Years or Older in Taiwan. PLoS ONE, 9(8). doi: 10.1371/journal.pone.0104945

13. Scott, W. B., Oursler, K. K., Katzel, L. I., Ryan, A. S., \& Russ, D. W. (2007). Central activation, muscle performance, and physical function in men infected with human immunodeficiency virus. Muscle \& Nerve, 36(3), 374-383. doi: 10.1002/mus.20832

14. Tran, T., Guardigni, V., Pencina, K. M., Amato, A. A., Floyd, M., Brawley, B., ... Montano, M. (2017). Atypical Skeletal Muscle Profiles in Human Immunodeficiency Virus-Infected Asymptomatic Middle-Aged Adults. Clinical Infectious Diseases, 66(12), 1918-1927. doi: 10.1093/cid/cix1121

15. Raad, J. (2014). Rehab Measures: Romberg test. (2014).

16. Whitney, S.L., Wrisley, D.M., Marchetti, G.F,, Gee, M.A., Redfern, M.S., \& Furman, J.M. (2005). Clinical measurement of sit-to-stand performance in people with balance disorders: validity of data for the Five-Times-Sit-to-Stand Test. Phys Ther. Oct;85(10):1034-45.

17. Powell, L. E., \& Myers, A. M. (1995). The Activities-specific Balance Confidence (ABC) Scale. The Journals of Gerontology Series A: Biological Sciences and Medical Sciences,50A(1). doi:10.1093/gerona/50a.1.m28

18. Erlandson, K., Plankey, M., Springer, G., Cohen, H., Cox, C., Hoffman, H., ... Brown, T. (2016). Fall frequency and associated factors among men and women with or at risk for HIV infection. HIV Medicine, 17(10), 740-748. doi: 10.1111/hiv.12378 
19. Sullivan, E. V., Rosenbloom, M. J., Rohlfing, T., Kemper, C. A., Deresinski, S., \& Pfefferbaum, A. (2010). Pontocerebellar contribution to postural instability and psychomotor slowing in HIV infection without dementia. Brain Imaging and Behavior, 5(1), 12-24. doi: 10.1007/s11682-010-9107-y

20. Ruiz, M. A., Reske, T. A., Cefalu, C. A., \& Estrada, J. A. (2013). Falls in HIV-Infected Patients: A Geriatric Syndrome in a Susceptible Population. Journal of the International Association of Providers of AIDS Care (JIAPAC), 12(4), 266-269. doi: 10.1177/2325957413488204

21. Prior, D. E., Song, N., \& Cohen, J. A. (2018). Neuromuscular diseases associated with Human Immunodeficiency Virus infection. Journal of the Neurological Sciences, 387, 27-36. doi: 10.1016/j.jns.2018.01.016

22. Bernard, C., Dilharreguy, B., Allard, M., Amieva, H., Bonnet, F., Dauchy, F., ... Catheline, G. (2013). Muscular Weakness in Individuals with HIV Associated with a Disorganization of the Cortico-Spinal Tract: A Multi-Modal MRI Investigation. PLoS ONE, 8(7). doi: 10.1371/journal.pone.0066810
23. Trenkwalder, C., Straube, A., Paulus, W., Krafczyk, S., Schielke, E., \& Einhäupl, K. (1992). Postural imbalance: An early sign in HIV-1 infected patients. European Archives of Psychiatry and Clinical Neuroscience., 241(5), 267-272.

24. Unhjem, R., Nygård, M., Hoven, L. T. V. D., Sidhu, S. K., Hoff, J., $\&$ Wang, E. (2016). Lifelong strength training mitigates the agerelated decline in efferent drive. Journal of Applied Physiology, 121(2), 415-423. doi: 10.1152/japplphysiol.00117.2016

25. Hinkin, C. H., Castellon, S. A., \& Hardy, D. J. (2000). Dual Task Performance in HIV-1 Infection. Journal of Clinical and Experimental Neuropsychology, 22(1), 16-24. doi: 10.1076/1380-3395(200002)22:1;1-8;ft016 